\title{
BASES PARA UN MODELO DE GUÍA PARA LA TRANSFORMACIÓN DE LAS IDENTIDADES DOCENTES UNIVERSITARIAS
}

\section{BASES FOR A GUIDING MODEL FOR THE TRANSFORMATION OF UNIVERSITY TEACHING IDENTITIES}

\section{Israel Alonso-Saéz, Aitor Garagarza}

\section{Karmele Artetxe y Nekane Beloki}

Universidad del Pais Vasco/ Euskal Herriko

Unibertsitatea

Universidad del País Vasco, UPV/EHU)

España

\section{RESUMEN}

Este trabajo repasa los diferentes marcos teóricos y conceptuales desde los que se han desarrollado investigaciones y aproximaciones a la identidad académica, para a continuación señalar los elementos claves a partir de la literatura existente que nos acerquen a un abordaje integral y holístico de lo que supone ser profesor/a de universidad hoy. A partir de esta revisión y elementos se propone una definición de la identidad docente universitaria. Tambiénse se profundiza en cada uno de estos aspectos para poner las bases de un modelo que sirva no solo para comprender la identidad académica, sino también como referencia a las actuaciones necesarias para su transformación en relación a una mejora e impacto positivo en su trabajo y entorno profesional.

PALABRAS CLAVE: Identidad docente universitaria, formación, cultura profesional, identidad académica, educación superior 


\section{ABSTRACT}

This work reviews the different theoretical and conceptual frameworks from which researches and approaches to academic identity have been developed, to then point out the key elements from the existing literature that bring us closer to an holistic and integral approach to what it means to be university professor today.From this review and elements a definition of university teaching identity is proposed. It also deepens in each of these aspects to lay the foundations of a model that serves not only to understand the academic identity, but also as a reference to the necessary actions for its transformation in relation to an improvement and positive impact on their work and environment professional.

University teaching identity, training, professional culture, academic identity, higher education.

\section{INTRODUCCIÓN}

\section{La identidad académica hoy}

No es fácil entender o comprender de qué hablamos cuando nos referimos a la identidad académica o docente universitaria. Cuando se citao se pregunta por ella, todo académico entiende de qué se está hablando. Forma parte de un conocimiento cultural en su relación con quién soy y quiénes somos como profesionales de la Educación Superior (ES). Es algo que instintivamente todos percibimos y que tiene que ver con la necesidad de pertenecer $y$ también de diferenciarnos. Pero si vamos más allá la cuestión se complica. ¿De qué estamos hablando?, ¿de la identidad relacionada con algo que está, se tiene o que se va construyendo?, ¿de una identidad individual o colectiva? La identidad viene dada por lo que nos ha tocado vivir y los recursos y espacios en los que nos hemos socializado profesionalmente pero, por otro lado, existe una sensación de ser guías y sujetos activos en su construcción; tiene que ver con lo que nos define, con la necesaria pertenencia (a una Facultad, departamento, disciplina,...), pero, también, a una necesidad de diferenciarnos y cuestionarnos las identidades dadas. No sólo está relacionada con lo que somos, sino también con lo que queremos llegar a ser. $Y$ ¿tenemos sólo una identidad? ¿Varias? ¿Cómo operan en diferentes espacios del trabajo académico? ¿La identidad sólo está relacionada con lo que pensamos sobre lo que somos, o también en lo que hacemos?

Podemos resumir, que si a cualquier persona - grupo, se les pregunta por la identidad académica como concepto, idea o proceso todo el mundo va entender de qué se está hablando, pero si se profundiza la confusión y dispersión de ideas, percepciones y vivencias puede ser muy grande.

Gewerc (2011) concluye que la identidad se percibe como algo evasivo y resbaladizo.

Es difícil acercarse a ella y cuando creemos que lo tenemos, se nos vuelve a escapar. Porque siempre está en proceso, es devenir y fácilmente puede confundirse haciendo alusión a otras cuestiones (...). El problema estriba quizás en que más que una cuestión que hay que revelar y describir, como algo que ya está dado, que existe, hay que considerarlo como (..) algo que se atrapa cuando se está construyendo, cuando reflexionamos y narramos nuestra historia (p.210).

En este sentido Nevgi y Löfström (2014) citan cómo Akkerman y Meijer (2011) plantean que la identidad docente se construye a través de posiciones del yo que están culturalmente mediadas, preservando la continuidad, pero a la vez en permanente cambio. Para estos autores/ as describirla supone navegar a través de características paradójicas, como la unidad y la multiplicidad, o la continuidad y discontinuidad, que hacen difícil de capturar y examinar su naturaleza y desarrollo.

Junto a esta complejidad y dificultad en su abordaje nos encontramos con un gran desarrollo en las últimas dos décadas de este 
ámbito de estudio. Hoy éste es un campo de investigación que está arrojando cada vez más datos y conocimientos, cuando hasta hace tres décadas no había tenido apenas atención por parte de investigadores y teóricos. Como se observa a continuación, distintas aproximaciones en todos los ámbitos de la geografía mundial han puesto de manifiesto la importancia de entender la naturaleza y dinámica de esta identidad académica en el desarrollo de la ES, y también, la necesidad de intervenir en ella para una mejora y desarrollo profesional que tenga impacto en la calidad y mejora en la labor de la ES.

Acercarnos a la identidad docente universitaria conlleva, por un lado, una necesaria aproximación global en la que aspectos de la identidad personal, profesional y cultural de cada docente entran en juego (Clegg,2008; Skerrett, 2008; Sheridan, 2013), y por otro, entenderla como una identidad profesional, en la medida que conlleva una serie de representaciones, roles, conocimientos y valores que comparte cada académico con profesionales de este ámbito educativo en el contexto local y global (Clarke, Hyde \& Drennan, 2013; Weise y Sanchez, 2013; Lewis, 2014). Esta identidad profesional parte de entender la labor del profesorado universitario como una profesión, en la medida que reúne una serie de condiciones que los cualifican y diferencian del resto de trabajadores. En palabras de Zabalza (2009):

Poseen una elevada formación (acreditada mediante un título); están integrados en una estructura organizativa propia de la profesión (un colegio profesional, un colectivo identificado como grupo de especialistas, etc.); son reconocidos socialmente como personas que saben de algo en especial siendo ese algo valioso para la sociedad; mantienen un compromiso con su formación permanente al socaire del desarrollo de su especialidad y poseen una elevada autonomía para ejercer su actividad profesional (se confía en que posean un buen "criterio profesional" basado en la formación recibida). (p.75)

Este mismo autor (2009) también declara que estas características provienen de una cultura profesional que está cambiando en el nuevo contexto del mercado de trabajo. En este sentido, hoy el abordaje del profesionalismo y las identidades profesionales supone hacerlo desde un nuevo escenario en el que anteriores seguridades y limitaciones claras de lo que suponía ser profesional en una disciplina, se han transformado en flexibilidad y difuminación de las fronteras de éstas (Trede, Macklin y Bridges, 2012; Lewis,2014). Como indica Bauman (2009), la construcción o la captura de una identidad, en este caso profesional, se ha convertido en un problema, porque su desarrollo es resbaladizo, flexible y siempre está en movimiento. El personal profesional tiene que adaptarse continuamente a un mercado laboral en evolución permanente. Para este autor (2009) el dilema que atormenta a los hombres y mujeres en el cambio de siglo no es tanto la forma de obtener la identidad profesional de su elección, como ha sido reconocida por las generaciones anteriores, sino saber qué identidad elegir y cómo mantenerse alerta y vigilante para realizar otra elección, en caso de que la identidad elegida previamente se retira del mercado o sea despojada de su poder de seducción.

Si el proceso anteriormente citado es global y se está produciendo en todas las profesiones, en el mundo de la ES está teniendo una especial incidencia (Barnett, 2002). De unas identidades profesionales en la ES bien delimitadas, con unos valores relacionados con el conocimiento, el pensamiento crítico y el servicio a la sociedad, se ha pasado a un nuevo escenario en el que estas identidades comparten y, en ocasiones, entran en colisión con los valores de rentabilidad, 
impacto económico y de mercado (Barnett, 2011; Winter y O,Donohue, 2012).

Dentro de las dos aproximaciones anteriormente descritas se encuentran una diversidad de enfoques y abordajes. En una revisión de diferentes investigaciones en todos los niveles educativos, Monereo y Pozo (2011), apoyándose en otros autores (Rocioeur, 1996; Roberts,2007), muestran una heterogeneidad de perspectivas teóricas desde las que se ha estudiado la identidad docente. Aproximaciones teóricas que se insertan en dos coordenadas analíticas: la naturaleza de lo que se construye (estructural y/o procesual) y los mecanismos (interpsicológicos o intrapsicológicos) a través de los que se construye y se puede inducir el cambio. Estos autores (2011), refiriéndose a este mapa de abordajes de la identidad, explican que aunque ningún estudio adopta uno de esos enfoques en estado puro todos intentan un mestizaje. También que aprecen dos grandes bloques teóricos y al estudio de la identidad. Un primer "acercamiento multi-representacional, mediadas por los procesos de autorregulación de las distintas identidades cognitivas situadas y una concepción de la identidad cultural y comunitaria, construida en términos de narrativas socialmente mediadas". (p.24)

\section{DIFERENTES APROXIMACIONES}

\section{TEÓRICAS Y METODOLÓGICAS EN EL} ESTUDIO DE LA IDENTIDAD DOCENTE

En este apartado vamos a realizar un recorrido por diferentes perspectivas de tipo sociológico, psicosocial y narrativo desde las que se han abordado las investigaciones en este campo.

Aproximaciones desde la perspectiva sociológica

En la búsqueda de un marco teórico que sustente el abordaje de la identidad docente y cómo opera ésta, son muchos los y las autoras (Gohier, Anadon, Bouchard, Charboonneau y Chevrier, 2001; Bolivar et al., 2005; Vaillant,2007; Avalos, Cavada, Pardo y Sotomayor, 2011; Gewerc,2011) que han adoptado la obra del sociólogo francés Dubar como referente. Este autor (1991) se acercó al estudio de la identidad desde la sociología de las profesiones y definió ésta como: “ (..) el resultado, a la vez estable y provisorio, individual y colectivo, subjetivo y objetivo, biográfico y estructural, de diversos procesos de socialización que conjuntamente construyen los individuos y definen las instituciones"( $p$. 111). Esta definición, que alumbra el abordaje de la identidad desde una perspectiva compleja, la caracteriza en una doble transacción: la identidad es una identidad para sí, pero también es una identidad para las demás personas (Dubar,2002). Tomando como punto de partida a este autor, Bolivar y colaboradores/as (2005) plantean la identidad del profesorado y sus modos de identificación como algo variable a lo largo de la historia personal y colectiva, tanto en las identificaciones que formulan los otros ("identidad por otro"), como de las que asume el propio sujeto ("identidad para si"). Y añade que en esta interacción entre estas dos atribuciones de identidad (convergencia/divergencia) se juega la "forma identitaria" que adquiere un grupo profesional, en este caso el personal docente universitario, y que esta articulación se produce en una tensión permanente entre la continuidad y la ruptura.

Si bien en esta aproximación se reconoce el aspecto personal y de interacción en la construcción de la identidad, para Dubar (1991) las identidades sociales y profesionales típicas no son ni expresiones psicológicas de personalidades individuales, ni el producto de estructuras sociales globales, sino construcciones sociales entre la interacción de las trayectorias individuales y los sistemas de empleo, trabajo y de formación: "productos de procesos de socialización, las identidades constituyen formas sociales de construcción individual de cada generación en cada sociedad." (Dubar,1991, p.262). Este hecho es matizado por Gewerc (2011) argumentando que 
"esta identidad es inacabada, entendida más como un devenir que un ser, se constituye en la representación, en cómo nos presentamos a nosotros mismos" (p. 198).

Uno de los autores influyentes en la teoría de Dubar, es el también sociólogo francés Bourdieu. Este autor (1998) es también citado en diferentes investigaciones relacionadas con el desarrollo del proceso identitario dentro de organizaciones como la ES (Gewerc, 1998; Gonzalez, 2003). En todas ellas se invoca el concepto que este autor denominó habitus y describió cómo el proceso de convertirse en profesor, asumir la profesión como propia, aprender sus reglas básicas de pertenencia e incorporar el saber y el saber hacer implica una construcción identitaria que no se produce de repente, sino que es un proceso.

Dentro de esta aproximación teórica a la identidad que pone el énfasis en la importancia del entorno cultural y socializador de la persona docente, Pozo y Monereo (2011) diferencian dos perspectivas teóricas. 1) La identidad como patrón cultural y 2) la identidad comunitaria y cultural. La primera concibe la identidad (self) como un patrón de respuestas o prácticas, culturalmente definidas, que se activa, de forma automática, ante determinadas condiciones sociales (Rogoff, 2003). La segunda, pone el énfasis en las instituciones educativas, como una subcultura que responde a determinados intereses, códigos, normas y rituales que promueven la construcción y el desarrollo de determinadas identidades en detrimento de otras. Según Pozo y Monereo (2011, p. 15-16)

Los individuos construyen sus identidades a través de la participación en las prácticas de distintas comunidades y de la generalización del conocimiento adquirido, de unos a otros contextos. Ser miembro de una comunidad educativa supone apropiarse de las prácticas específicas que son importantes en su seno. A medida que se aprende se cambia la participación en las prácticas comunitarias $\mathrm{y}$, a un tiempo, la identidad como miembro de esa comunidad (de los roles periféricos a los expertos) (..) dicho de otro modo, se aprende a ser profesor o alumno en esa institución.

\section{APROXIMACIONES DESDE LA PERSPECTIVA PSICOSOCIAL}

En diferentes abordajes de la identidad docente es habitual centrar el análisis en las representaciones sociales (Mazzitelli, Aguilar, Guirado, Olivera, 2009). Moscovici (1979) define las representaciones sociales como construcciones simbólicas que se originan en la interacción social y se recrean a lo largo del tiempo. Son de orden cognitivo, dado que las personas no son pasivas frente a la información que reciben del grupo, sino que construyen significados y teorías sobre la realidad, en una vinculación dialéctica entre lo individual y lo social.

Este estudio de las representaciones sociales nos permite adentrarnos en la forma en que los sujetos interpretan y construyen su conocimiento sobre la realidad y en los modos en que este conocimiento influye en sus comportamientos y actitudes frente a los problemas de la vida cotidiana (Mazzitelli et al, 2009), en este caso de la praxis docente. Un aspecto útil para el abordaje de la identidad docente ${ }^{1}$ es señalar cómo estas representaciones sociales constituyen un todo estructurado y organizado compuesto por un conjunto de informaciones, creencias, opiniones y actitudes referidas a un objeto que están jerarquizadas y organizadas alrededor de un núcleo central y otro periférico (Abric, 2001). Como explican Mazzitelli et al (2009)

(...) están organizados alrededor de un núcleo central conformado por algunos

$1 \quad$ A este respecto Mazziteli et al (2009) plantean que el estudio de las representaciones sociales de los profesores sobre la docencia y sobre el ser un mejor docente posibilitaría encontrar los rasgos que aportan a la especificidad de este campo profesional, con sus diversos matices. Es decir, los elementos que permiten a los docentes reconocerse y ser reconocidos como tales. 
elementos que otorgan una significación particular a la representación. En el núcleo, la estabilidad de los elementos garantiza la permanencia y el carácter innegociable de la representación. Estas creencias, opiniones y actitudes están ligadas generalmente a la memoria e historia grupal $\mathrm{y}$, por lo tanto, ofrecen una gran resistencia a las transformaciones del contexto social. El núcleo central cumple con dos funciones: por un lado, otorgar el significado a la representación; por otro, organizar el resto de los elementos. También encontramos un sistema periférico que tiende a preservar el núcleo de posibles transformaciones, y que es flexible y variable debido a que es más sensible al entorno inmediato. (p. 269)

Otro marco teórico con gran influencia en el estudio de la identidad en el ámbito educativo (Gohier et al,2001; González, 2003; Lopes, 2007; Monereo y Pozo,2011a,) es el del interaccionismo simbólico. Esta teoría desarrollada por Mead (1934), en la década de los años 30, responde a un debate existente en aquel momento histórico relacionado con el estudio de los fenómenos sociales y vitales desde el prisma de la subjetividad o del estructuralismo. Este autor plantea que el desarrollo de la identidad personal es fruto de la internalización de la perspectiva de los y las demás sobre uno o una misma, mediante la interacción y la experiencia social; es decir, la interacción recíproca entre la sociedad y el self (sí mismo). De esta manera, desde esta corriente, ni la sociedad humana podría existir sin mentes y sí-mismos, ni las mentes y selfs podrían surgir sin la sociedad humana (Uriz, 1993 en Gonzalez, 2003). Como describe Sciolla (1983) la identidad tiene un carácter inter-subjetivo y relacional. Esto significa que es el resultado de un proceso social ya que surge y se desarrolla en la interacción cotidiana con los otros y el medio. Esto permite que el individuo se reconoca a sí mismo, reconociéndose en los otros.

Este carácter interactivo y relacional, así como la importancia de los y las otras en el desarrollo de la identidad es el gran aporte del interaccionismo simbólico.

Desde otras corrientes psicológicas también se han realizado aportaciones importantes al abordaje de la identidad. Por ejemplo, la aportación de la psicología humanista en la importancia de la conciencia y la autorrealización, así como de las crisis en el desarrollo de la identidad personal docente (Gohier et al, 2001). En esta línea, Day (2006) ha vuelto a poner el énfasis en el valor de estos aspectos de autorrealización profesional en la identidad del profesorado planteando que un aprendizaje y una enseñanza eficaz sólo son posibles si se basan en el ejercicio de la pasión de los educadores y educadoras por enseñar.

Dentro de la psicología, también en las últimas dos décadas, desde las denominadas teorías realistas y desde la psicología cognitiva se ha puesto de relieve la importancia que las teorías implícitas e inconscientes del personal docente tiene en su desarrollo identitario. De esta manera, se sitúan dentro del núcleo duro de las creencias e identidad docente (Herrán, 2009; Korthagen, 2010; Pozo, 2011; Gómez,, 2011). Como plantea Korthagen (2010):

Las barreras entre el ámbito profesional y ámbito personal se difuminan en la vida práctica de los y las docentes, pues sus decisiones no se sustentan ni sólo ni prioritariamente en su conocimiento explícito profesional, sino de forma muy decisiva en sus modos de pensar, sentir y actuar, en sus plataformas de creencias y hábitos, la mayoría de ellos implícitos e inconscientes, que constituyen su peculiar personalidad (Korthagen, 2000 en Gómez, 2011, p. 22). 
También en el campo del estudio de la identidad desde el campo psicológico ha surgido un debate sobre si la identidad es única y cerrada o múltiple y compleja. Como plantea Pozo (2011), después de fuertes debates teóricos, hoy se acepta la pluralidad de formatos y sistemas representacionales en la mente humana, siendo el reto actual "darle un sentido teórico, mediante una integración de esas múltiples identidades que, al tiempo que preserve esa diversidad conquistada o ganada (..) permita construir algún tipo de unidad o integración en esas múltiples diversidades" (p. 48). Pozo y Monereo (2011, p. $60)$ lo expresan de la siguiente manera:

La posibilidad de ser uno y muchos o, dicho de otro modo, de salvaguardar una "identidad" que ofrezca estabilidad y permanencia a nuestro modo de "ser profesor" y, al mismo tiempo, que permita desarrollar versiones de ese ser profesor para adaptarnos a las demandas cambiantes de cada contexto educativo (..)

Algunas teorías psicológicas, como el "dialogic self" (Branco y Madureira, 2008; Akerman y Meijer,2011), han planteado que las diferentes identidades de cada persona entran en un diálogo para adaptarse a cada contexto concreto de actuación adoptando una u otra. Pozo (2011), sin embargo, critica esta perspectiva, ya que, en su opinión, es reduccionista y para este autor es necesario plantear cómo esa diversidad de identidades interrelaciona y confluye en una integración jerárquica en que la persona interactúa con un medio siempre complejo.

Ese yo indivisible - como estado mental unitario - tendría por función precisamente la reconciliación integradora, que en lugar de ejercerse por la vía baja del contexto - un solo yo diferente para cada situación- se debe ejercer por la vía alta de la explicitación progresiva (..). Las diversas identidades deberían tender a encajarse o integrarse unas en otras, $\mathrm{o}$ al menos dialogar entre sí, en busca de esa integración (Pozo,2011, p. 52).

Para este autor (2011) esta integración no sería estática y estaría relacionada con la conciencia del aquí y el ahora, como un estado subjetivo y consciente indivisible pero discontinuo, sería la forma más simple de vivir y sentir la identidad, un yo plenamente presente, aquí y ahora. Sería la conciencia central, en el sentido de Damasio (1999).

\section{APROXIMACIONES DESDE LA PERSPECTIVA NARRATIVA}

Otro enfoque teórico, proveniente de otras disciplinas como la filosofía y la antropología, muy influyente en este campo en la actualidad es el narrativo. Son muchos los estudios sobre la identidad docente que tienen como base teórica o metodológica esta perspectiva (Gewerc, 2001; Bolívar et al,2004; Vaillant, 2007, Lauelo y Crespo, 2011; Richmond, Juzwik y Steele, 2011;;). Este enfoque plantea acercarnos a la identidad como una narración autobiográfica que emerge de las transacciones que se producen en un determinado contexto. Bolívar et al. (2005) plantean que las personas construyen su identidad individual haciendo un autorrelato, que no es sólo recuerdo del pasado, sino un modo de recrearlo en un intento de descubrir un sentido e inventar el yo, que pueda ser socialmente reconocible. Citan a Ricoeur (1996), uno de los autores de referencia desde este enfoque, para resaltar la centralidad del relato en la constitución identitaria:

La persona, entendida como personaje de relato, no es una identidad distinta de 'sus' experiencias. Muy al contrario: comparte el régimen de la identidad dinámica propia de la historia narrada. El relato construye la identidad del personaje, que podemos llamar su identidad narrativa, al construir la de la historia narrada. Es la identidad de 
la historia la que hace la identidad del personaje (p.147).

En este sentido Gewerc (2011) plantea que la identidad surge:

cuando se está construyendo, cuando reflexionamos y narramos nuestra historia. Cuando hacemos el esfuerzo de reconstruirla a través del relato la identidad cobra vida y da sentido a esa vida. Como dice Ricoeur La identidad se constituye por el sujeto al que el relato asigna una identidad narrativa (p. 210).

\section{PLANTEANDO LAS BASES PARA LA CONSTRUCCIÓN DE UN MODELO HOLÍSTICO PARA LA TRANSFORMACIÓN DE LAS IDENTIDADES DOCENTES UNIVERSITARIAS}

Una de las conclusiones del repaso histórico anterior sobre los marcos teóricos desde los que se está abordando el estudio de la identidad docente coincide con la idea de que no parece que se necesiten más definiciones o referentes teóricos, sino que hace falta un intento de integración, para conocer cómo interactúa en diferentes entornos específicos, como plantean Falsafi y Coll (2011):

(...) el intento por comprender cómo se construyen las identidades y cuáles son sus funciones es un intento por comprender cómo las personas se ven y se definen a sí mismas en relación con los otros y con su entorno. (...) pero la convicción de que lo que necesitamos en el momento actual no son nuevas definiciones de la identidad y de las identidades. A nuestro juicio, la prioridad debe ser conjuntar las múltiples y diversas piezas teóricas y metodológicas aportadas por la investigación, con el fin de resolver el puzzle de la construcción de las identidades y poder hacer un uso consciente del conocimiento de ese proceso de construcción (p. 97).

En este sentido, investigar y acercarnos a la identidad docente universitaria plantea una importante dicotomía entre seguir un enfoque concreto desde el cual hacer su abordaje o intentar buscar un modelo integral y holístico que recoja las diferentes perspectivas que se han ido citando. En este trabajo finalizamos con el intento de avanzar hacia la segunda opción. La tentativa de buscar y comenzar a construir las bases de una propuesta teórica que, desde una visión global, recoja los diferentes aspectos que están relacionados con la construcción y cambios identitarios docentes en procesos de innovación curricular en la ES. Para ello, a partir de la revisión teórica y bibliográfica realizada por Alonso, Lobato y Arandia (2015), se proponen diferentes dimensiones para la construcción de un modelo global y holístico que permita abordar las identidades docentes en procesos de cambio. En este sentido definimos la identidad docente universitaria como:

\begin{tabular}{|c|}
\hline $\begin{array}{l}\text { Un proceso dinámico, complejo y } \\
\text { siempre emergente; }\end{array}$ \\
\hline $\begin{array}{l}\text { Construido a partir de las } \\
\text { representaciones, } \\
\text { experiencias, sentimientos y significados } \\
\text { que se han ido desarrollando a lo largo } \\
\text { de su trayectoria profesional y vital; }\end{array}$ \\
\hline $\begin{array}{l}\text { Que le permiten en cada momento } \\
\text { concreto, de un manera dialógica, } \\
\text { integrada y contextualizada, afrontar } \\
\text { y responder a las situaciones que } \\
\text { aparecen en su quehacer docente y } \\
\text { académico; }\end{array}$ \\
\hline
\end{tabular}

Se desarrolla y construye siempre en un contexto cultural, profesional y ético. 
Como se observa en la figura 1 los cuatro aspectos nucleares en la construcción de este modelo para la comprensión de la construcción identitaria del profesorado universitario son:

- las representaciones biográficas y sociales sobre lo que significa ser profesor/a de universidad. Unas representaciones que las personas docentes van construyendo a partir de su historia y experiencia personal y profesional y que, a su vez, recibe del contexto profesional en el que socializa (Kreber, 2010; Weise y Sánchez, 2013). Estas representaciones son de diferentes tipos, teniendo gran importancia las relativas al rol profesional: cómo entiende y dónde pone el énfasis en relación con los diferentes roles relacionados con la docencia, la investigación y la gestión presentes en la identidad académica. Así mismo, también juegan un papel importante, en este caso dentro del rol relativo a la docencia, las representaciones que el o la docente tenga del proceso de enseñanza y aprendizaje y las emociones y sentimientos relativas a ella (Monereo y Badia, 2011).

- La dimensión ética y los valores. Esta dimensión está relacionada con la construcción personal y con una referencia más amplia de un marco de valores vinculados con el bien común, la conciencia crítica, el respeto y el compromiso con la mejora y el cambio entre otros (Clarke, Hyde y Drennan, 2013; Fitzmaurice, 2013). Se desarrolla a lo largo de la vida profesional de cada docente teniendo incidencia en su identidad. Hoy en día nos encontramos ante un proceso conflictio de integración entre los valores tradicionales de la academia (libertad, búsqueda de la verdad, autonomía y servicio) con los valores que surgen de una cultura mercantilista y neo-liberal en la ES relacionados con la competitividad, el monetarismo y la auditoría (Winter y
O,Donohue, 2012).

- Las interacciones y diálogo. Como plantean Leibowitz y colaboradores (2014) esta dimensión muestra la importancia que tienen en la construcción y transformación identitaria las interacciones con las personas que comparten espacios profesionales. Esta interacción posibilita los procesos de reconocimiento, construcción y reconstrucción identitaria e identificación y diferenciación. A través del contacto con las personas afines en sus diferentes espacios (disciplinas, departamentos, centros, grupos docentes, investigaciones,..) y también con el alumnado y el personal profesional, cada académico construye su identidad a partir de las comunidades de práctica en la que se inserta, y a su vez, la modifica (Caballero y Bolívar, 2015).

- Conocimientos y saberes. En el corazón de la identidad académica se encuentra ser una referencia en el conocimiento sobre la disciplina que investiga y enseña. Tener competencia en su dominio, así como en posibilitar su aprendizaje. También tienen importancia aquellos conocimientos sobre el "ser" y "estar" que le permiten al docente interactuar y desenvolverse en su labor profesional (Caballero y Bolívar, 2015). Saberes relacionados con lo disciplinar, pero también con lo interdisciplinar, en una labor académica cada vez más compleja y cuyas referencias de conocimiento necesariamente implican diferentes abordajes y referencias teóricas y prácticas.

A estos cuatro elementos hay que añadirles otros procesos que se dan a un nivel externo y personal. Por un lado, factores relacionados con la cultura universitaria global y la relativa al contexto local en el que se desarrolla, suponen el marco en el que los elementos anteriormente citados se desenvuelven (Rivas, Sepúlveda y Rodrigo, 2005; Tryggvason, 2012. Por otro 
lado, este proceso simultáneo y global necesita que cada docente lo integre. Cada académico teniendo en cuenta una necesaria flexibilidad y estabilidad integra de manera jerarquizada y contextualizada los anteriores aspectos para responder a los retos y necesidades de cada una de las acciones y tareas que lleva a cabo en su día a día profesional (Pozo, 2011). En esta integración juegan un papel importante los elementos cognitivo-emocionales, así como el carácter implícito de muchos de los procesos (Korhonen y Törmä, 2014) y que permiten este ejercicio permanente de integración.

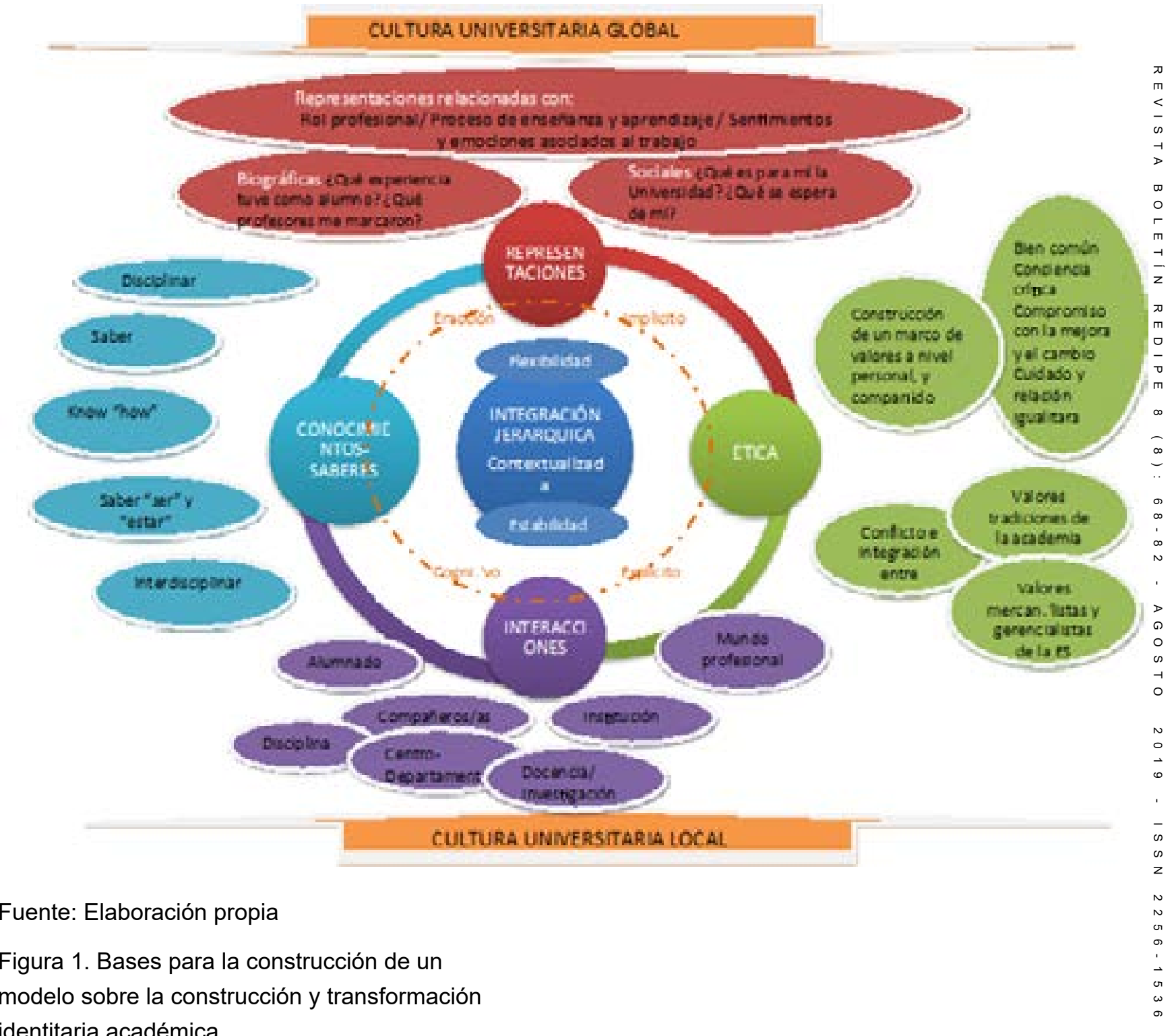

Las bases anteriormente descritas para avanzar en la construcción de un modelo holístico relacionado con la construcción y transformación de las identidades académicas tiene a nuestro entender una doble virtualidad:
- En primer lugar, ayudar a comprender este fenómeno. Nos permite ver los límites a los abordajes realizados sólo desde un enfoque. Aunque necesarios y útiles para la construcción de conocimiento en este ámbito dejan necesariamente fuera 
aspectos importantes a la hora de intervenir en estos procesos.

- En segundo lugar, servir de guía para la intervención y la transformación en estos procesos identitarios, de manera que pueda ayudar a instituciones académicas, grupos de docentes o a docentes, individualmente, a trabajar e incidir en un aspecto importante para el cambio educativo, en este caso en la ES, como es la identidad docente.

Para finalizar, apuntamos algunas cuestiones y aspectos a tener en cuenta a la hora de trabajar con este modelo:

1. La identidad docente, su cambio y transformación, es una de las claves y pilares a la hora de abordar cambios educativos profundos y sostenibles. Si no cambian las percepciones y el enfoque de la tarea a realizar por cada docente, difícilmente cambiará la realidad, por mucho que desde otras instancias institucionales se impulse o explicite cómo y de qué manera se debe llevar a cabo este cambio (Monereo y Pozo, 2011). Este modelo que se presenta puede ayudar a entender que cualquier intento en esta dirección debe tener en cuenta y trabajar con diferentes dimensiones y aspectos que están detrás de estos procesos. Es importante tener en cuenta actuaciones que conjuguen lo global y lo cercano a la realidad que vive el o la docente, que tenga en cuenta el desarrollo institucional que se quiere impulsar, pero también la rama disciplinar o la cultura docente concreta del centro en la que se quiere llevar a cabo. Con sólo movimientos en algunos de estos niveles sin tener en cuenta la globalidad difícilmente se van a producir cambios profundos y sostenibles.

2. El papel relevante que tiene todo lo relacionado con la interacción y lo dialógico. Este aspecto interpersonal es fundamental y se va a dar y producir de todas formas, sea impulsado o no. Por ello, si de lo que se trata es de promover un cambio cultural, un tránsito en la identidad docente de toda una institución universitaria o parte de ella, parece imprescindible generar espacios relacionales donde se pueda intercambiar y discutir sobre estos procesos. Pero no sólo estaríamos hablando de lo formal, sino también de lo informal. En la medida que se posibilite una cultura docente de seguridad para abrir lo que cada uno y una hace, las dudas y cuestiones que aparecen, en definitiva, abrir las puertas a la intimidad que cada docente vive en su práctica profesional, estos podrán ver, saber, mostrar, afianzar o replantearse sus representaciones y prácticas.

3. La interacción no sólo con el personal afín de profesión, sino también con el alumnado y profesionales del área de conocimiento en el que desarrollan su trabajo. No olvidemos que, dicho de diferentes modos, a los dos pilares que esta nueva identidad docente universitaria debe de responder son una docencia centrada en el aprendizaje de los alumnos y alumnas; y su conexión con la sociedad donde se inserta y desarrolla. Por ello, resulta difícil imaginar un tránsito identitario docente en este sentido, sin ser compartido, discutido y contrastado con sus protagonistas, en este caso el alumnado y los agentes sociales relacionados con la materia que imparte o investiga.

4. Este proceso necesita de espacios de intercambio y de reflexión personal y grupal, como se planteaba anteriormente, y también de formación en esta dirección. Espacios de formación en los que se trabajen, de manera activa y experiencial, aspectos reflejados como las representaciones profundas, las emociones, los valores o el papel que juega lo implícito que, de otra manera, difícilmente van a hacerse conscientes y van a poder ser replanteados. Espacios que conjuguen 
modelos teóricos de hacía dónde se quiere transitar con la realidad y práctica que cada docente vive y reflexiona individual y colectivamente. Estos espacios formativos formales e informales también tienen que ayudar a que los y las docentes adquieran los conocimientos y las competencias que esta nueva identidad docente a la que se quiere transitar requieren: competencias docentes, relacionales o que unan la docencia y la investigación. Algunas de estas competencias muestran un escenario complejo y a profundizar (Caballero $y$ Bolivar, 2015). Cuestiones discutidas como el mantra actual que plantea la excelencia o la innovación en la ES sí que nos pueden ayudar a ver un horizonte en el que la motivación y la implicación de los y las docentes con la tarea que realizan, su mejora y su desarrollo resultan imprescindibles.

5. Estos tránsitos y cambios no son sencillos ni lineales. Necesitan de tiempo y requieren respetar los ritmos de una siempre diversidad de estilos e identidades docentes. Por lo que, exigen activar y generar acciones en todos los ámbitos del sistema, teniendo claro un horizonte institucional de hacia dónde se quiere transitar, y medios para poder llevarlo a cabo; asimismo, también exigen la implicación de cada docente y grupo de ellos en estos procesos. En definitiva, un necesario equilibrio entre una siempre rica diversidad dentro de los y las docentes, un proceso que sea discutido y construido colectivamente y una visión institucional fuerte que lo oriente. Esta es la base y la oportunidad para posibilitar tránsitos identitarios en los y las docentes que tengan un impacto positivo en la mejora de la labor educativa y social que realizan.

\section{REFERENCIAS BIBLIOGRÁFICAS}

Abric, J.(2001). Prácticas sociales y representaciones. Mexico: Coyoacán.

Akkerman, S. F., \& Meijer, P. C. (2011). A dialogical approach to conceptualizing teacher identity. Teaching and Teacher Education, 27(2), 308-319.

Alonso, I., Lobato, C., \& Arandia, M. (2015). La identidad profesional docente como clave para el cambio en la educación superior. Opción: Revista de Ciencias Humanas y Sociales, (5), 51-74.

Avalos, B., Cavada, P., Pardo., M. \& y Sotomayor, M (2010) La profesión docente: Temas y discusiones en la literatura internacional. Estudios Pedagógicos Vol. $36 \mathrm{n}^{\circ} 1$ (2010), 235-263.

Barnett, R. (2002). Claves para entender la universidad en una era de supercomplejidad. Girona: Pomares.

Barnett, R. (2011a). Lifewide education: A new and transformative concept for higher education. In Jackson, $\mathrm{N}$ (Ed.), Learning for a complex world: A lifewide concept of learning, education and personal (22-38). Bloomington: AuthorHouse

Bauman, Z. (2009). Identity in a globalizing world. In Identity in question, ed. A. Elliot and P. du Gay. London: Sage.

Bolívar, A., Fernandez, M., \& Molina E. (2005). Researching Teachers' Professional Identity: A Sequential Triangulation. In Forum Qualitative Sozialforschung/Forum: Qualitative Social Research (Vol. 6, No. 1). Consultado en http://www. qualitative-research.net/index.php/fqs/ article/view/516/1116 
Bourdieu, P. (1988) Cosas Dichas. Buenos Aires: Gedisa.

Branco, A., \& Madureira, A. F. (2008). Dialogical Self in action: The emergence of SelfPositions among complex emotional and cultural dimensions. Estudios de Psicología, 29(3), 319-332.

Caballero, K., \& Bolívar, A. (2015). Identidades profesionales, concepciones de enseñanza-aprendizaje $y$ estrategias docentes del profesorado universitario. REDU. Revista de Docencia Universitaria, 13(1), 57-77.

Clarke, M., Hyde, A., \& Drennan, J. (2013). Professional identity in higher education. In The academic profession in Europe: New tasks and new challenges (pp. 7-21). Springer Netherlands.

Clegg, S. (2008). Academic identities under threat?. British Educational Research Journal, 34(3), 329-345.

Damasio, A. (2001). La sensación de lo que ocurre: cuerpo y emoción en la construcción de la conciencia. Madrid: Debate.

Day, C. (2006). Pasión por enseñar: la identidad personal y profesional del docente y sus valores. Madrid: Narcea Ediciones.

Dubar, C. (1991). La socialisation. Construction des identités sociales et professionnelles. París: Armand Colin.

Dubar, C. (2002). La crisis de las identidades. La interpretación de una mutación. Barcelona: Bellaterra.

Falsafi, L. y Coll, C. (2011). La construcción de la identidad de aprendiz. Coordenadas espacio-temporales. En Monereo, C. y J.I. Pozo, J.I. (coord.). La Identidad en
Psicología de la Educación: necesidad, utilidad y límites (pp. 77-98) Madrid: Narcea

Fitzmaurice, M. (2013). Constructing professional identity as a new academic: a moral endeavour. Studies in Higher Education, 38(4), 613-622.

Gewerc, A.(2011). Identidades docentes en contextos turbulentos. Espacios, tiempos y afectos. C. Monereo y J. I. Pozo. La identidad en Psicología de la educación (pp. 189-212) Madrid: Narcea

Gohier, C., Anadón, M., Bouchard, Y., Charbonneau, B., \& Chevrier, J. (2001). La construction identitaire de l'enseignant sur le plan professionnel: un processus dynamique et interactif. Revue des sciences de l'éducation,27(1).

González, V. (2003). La profesionalidad del docente universitario desde una perspectiva humanista de la educación. Ponencia presentada en el I Congreso Iberoamericano de Formación de Profesores, celebrado en la Universidad Federal de Santa María, Rio Grande del Sur, Brasil, en abril de 2000. Disponible en http://www.udesarrollo.cl/ udd/CDD/articulo/files/Profesionalidad_ Docente_U niversitario.doc 13-05-14

Gómez, Á. (2010). Nuevas exigencias y escenarios para la profesión docente en la era de la información y de la incertidumbre. Revista Interuniversitaria de formación del Profesorado, (68), 1736

Herrán, A. (2009). Estadios de evolución docente. Tendencias pedagógicas, (14), 375-416. 
Korhonen, V., \& Törmä, S. (2014). Engagement with a teaching career-how a group of Finnish university teachers experience teacher identity and professional growth. Journal of Further and Higher Education, (ahead-of-print), 1-18.

Korthagen, F. (2010). La práctica, la teoría y la persona en la formación del profesorado. Revista Interuniversitaria de Formación del Profesorado, (68), 83-102.

Kreber, C. (2010) Academics' teacher identities, authenticity and pedagogy, Studies in Higher Education, 35:2, 171-194

Lauleza, J.L y Crespo, I. (2011). La "multiplicidad de voces" en la construcción de la identidad minoritaria. En Monereo, C. y J.I. Pozo, J.I. (coord.). La Identidad en Psicología de la Educación: necesidad, utilidad y límites (pp. 115-140) Madrid: Narcea

Leibowitz, B., Ndebele, C., \& Winberg, C. (2014). 'It's an amazing learning curve to be part of the project': exploring academic identity in collaborative research. Studies in Higher Education, 39(7), 1256-1269.

Lewis, K. (2014) Constructions of professional identity in a dynamic higher education sector, Perspectives: Policy and Practice. Higher Education, 18(2), 43-50

Lopes, A. (2007). La construcción de identidades docentes como constructor de estructura y dinámica sistémicas: argumentación y virtualidades teóricas y prácticas. Revista de currículum y formación del profesorado, 3, 1-25.

Mazitelli, C., Aguilar. S., Guirado, A. (2009) Representaciones sociales de los profesores sobre la dociencia: contenido y estructura. Revista de Educación,
Lectura y Sociedad, 6 (6), 265-290

Monereo, C y Pozo, J.I. (2011). La identidad en psicología de la educación. En Monereo, C. y Pozo, J.I. (coord.). La Identidad en Psicología de la Educación: necesidad, utilidad y límites (pp. 45-56) Madrid: Narcea

Monereo, C. y Badia, A. (2011). Los heterónimos del docente. Identidad, selfs y enseñanza. En Monereo, C. y J.I. Pozo, J.I. (coord.). La Identidad en Psicología de la Educación: necesidad, utilidad y límites (pp. 57-66) Madrid: Narcea

Moscovici, S. (1979) El psicoanálisis, su imagen y su público. Buenos Aires: Editorial Huemul

Nevgi, A., \& Löfström, E. (2014). Visualisations as a means for exploring academics' teacher identities. International Journal for Academic Development, 19(3), 174185.

Pozo, J.I. (2011). La construcción de la identidad en la Psicología cognitiva. Del aprendizaje situado a la integración jerárquica. En Monereo, C. y Pozo, J.I. (coord.). La Identidad en Psicología de la Educación: necesidad, utilidad y límites (pp. 45-56) Madrid: Narcea

Richmond, G., Juzwik, M. M., \& Steele, M. D. (2011). Trajectories of teacher identity development across institutional contexts: Constructing a narrative approach. Teachers College Record, 113(9), 1863-1905.

Ricoeur, Paul (1996) Sí mismo como otro. Madrid: Siglo XXI.

Rivas, J. I., Sepulveda, M. P., \& Rodrigo, P. (2005). La cultura profesional de los 
docentes en enseñanza secundaria: Un estudio biográfico. Archivos Analíticos de Políticas Educativas, 13(47), 1-26

Roberts, A. L. (2007). Relaciones entre identidad y aprendizaje. Cultura y Educación, 19 (4), 379-394.

Rogoff, B. (2003). The cultural nature of human development. Oxford: Oxford University Press.

Sciolla, L (1983): Identitá. Turín: Rosenberg and Sellier,

Sheridan, V (2013). A risky mingling: academic identity in relation to stories of the personal and professional self, Reflective Practice: International and Multidisciplinary Perspectives. 14, 4, pp568-579.

Skerrett, A. (2008). Biography, identity, and inquiry: The making of teacher, teacher educator, and researcher. Studying Teacher Education, 4(2), 143-156.

Trede, F., Macklin, R., \& Bridges, D. (2012). Professional identity development: a review of the higher education literature. Studies in Higher Education, 37(3), 365-384.

Tryggvason, M. T. (2012). Perceptions of identity among Finnish university-based subject teacher educators. European Journal of Teacher Education, 35(3), 289-303.

Vaillant, D. (2007). Mejorando la formación y el desarrollo profesional docente en Latinoamérica. Revista Pensamiento Educativo, 41(2), 207-222

Weise, C., \& Sànchez, S. (2013). Identidad docente y estrategias de resolución de incidentes críticos en contextos universitarios de alta diversidad sociocultural. Cultura y Educación, 25(4), 561-576.
Winter, R. P., \& O'Donohue, W. (2012). Academic identity tensions in the public university: which values really matter?. Journal of Higher Education Policy and Management, 34(6), 565-573

Zabalza, M. Á. (2009). Ser profesor universitario hoy. La Cuestión Universitaria, 5, 69-81 